\section{CIRDIS : A BASIC program for computing interindividual distances within a circular area}

\section{WAYNE P. ASPEY \\ Department of Psychology, Dartmouth College Hanover, New Hampshire 03755}

The use of interindividual distances as a quantitative measure of social attraction is widespread in animal social psychology (Barefoot, Aspey, \& Olson, 1975; Eckman, Meltzer, \& Latané, 1969; Latané, 1969; Latané, Cappell, \& Joy, 1970; Latané \& Glass, 1968; Latané, Schneider, Waring, \& Zweigenhaft, 1971). However, obtaining large numbers of accurate distance measurements can be unwieldy, especially when numerous subjects are observed over multiple trials and testing sessions. The program CIRDIS refers to "circle distances," and facilitates raw data collection and organization by computing interindividual distances between two freely behaving subjects in a circular open field over any number of trials and testing sessions.

The program is written for a circular area marked off into 49 numbered sectors of nearly equal area and shape by a series of concentric circles and radii (Figure 1). Field diameter is user specified, and the program is adaptable to a variety of applications wherever pairs of locations can be specified in terms of 49 sectors. Additional research examples, other than from rat

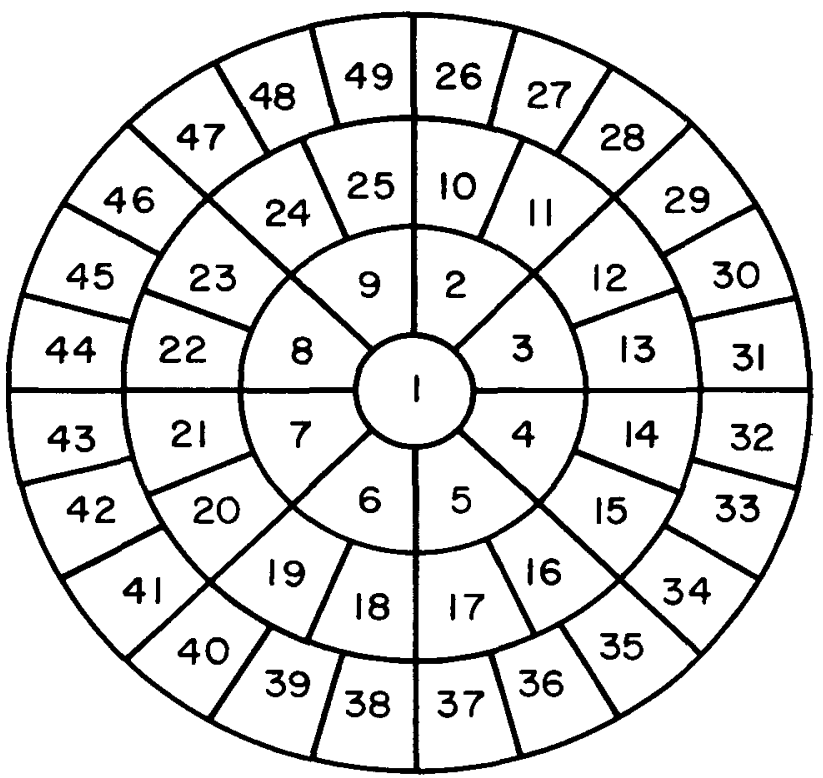

Figure 1. Diagram of the circular area on which the program CIRDIS is based; circle diameter is user specified. Interindividual distances are calculated from the center of the sector where one member of a pair is located to the center of the sector where the other member is located. social psychology, utilizing the program include distance calculations between prey and a spider on an orb web, and computation of personal space among groupdwelling spiders on a sheet-like web (Burgess, Note 1).

Diagrams of the numbered circular area (Figure 1) make convenient data sheets for recording subjects' locations during data collection or for transcribing tape recorded protocol. The program can be used to generate a theoretical chance interindividual distance by entering all combinations of sector numbers and obtaining the average of all possible distances. Furthermore, control distances can be computed by recording each partner's locations when tested alone and computing the distances as if the partners had been tested together. This measure provides an indication of the expected interindividual distance between partners if they were to move through the field independently of each other.

Description. After "location" is operationally defined (e.g., that sector where a rat's right forepaw is located during a given trial), the numbers of the sectors where each member of a pair is located are recorded. Interindividual distance is computed from the center of the sector where one partner is located to the center of the sector where the other partner is located. Userspecified parameters for each pair during one testing session include: (1) the number of data pairs (P) per trial, and (2) the number of trials (T) per testing session. Thus, total data pairs from one subject pair during one testing session is derived by PT. Corresponding sample data for one subject pair might include: four data pairs $(P=4$, one pair of locations recorded every $15 \mathrm{sec}$ during a 1 -min trial) recorded for $10 \mathrm{~min}(\mathrm{~T}=10$ trials) over seven testing sessions (e.g., days).

Input. Three empirically determined distances, user supplied at indicated points in the program, include the distance from the center of Sector 1 to the center of any other sector in: (1) the first, innermost concentric ring (Sectors 2-9); (2) the second, middlemost ring (Sectors 10-25); and, (3) the third, outermost ring (Sectors 26-49). In addition to user-supplied identifying labels, the program accepts any number of subject pairs and testing sessions. Input for each subject pair includes: (1) the number of data pairs per trial; (2) the number of trials per testing session; and, (3) the appropriate series of data pairs, entered as Sectors 149 , for a given testing session.

Output. In addition to user-supplied labels for individual subjects, groups, trials, and testing sessions, output from CIRDIS consists of: (1) the original sector entries, by pairs, with the accompanying distance for each pair on all trials; (2) means and standard deviations of all distances within each trial for each subject pair; and, (3) means and standard deviations of all trials within each testing session for every subject pair. 
Restrictions. The present program is limited to computing interindividual distances between subject pairs with the assumption that only two animals are interacting or being tested together at the same time. However, the program can be changed readily to accept trios, quartets, and so forth, of subjects by modifying the necessary number of loops and accumulation variables to accommodate the additional iterations. Under these circumstances, CIRDIS computes interindividual distances between all possible pair combinations of the trio, quartet, and so forth, interacting simulteneously.

Computer and language. The program CIRDIS is written in BASIC (Kemeny \& Kurtz, 1968; Sharpe, 1967), and was developed using the Dartmouth/GE-635 computing system at the Kiewit Computation Center (Nevison, 1976) and the time-sharing terminals in the Department of Psychology, Dartmouth College. CIRDIS was designed to be implemented on a variety of computer systems and has been used extensively on the IBM 360/44 computer, using the OUIJI system (acronym for "Ohio University Instructional Job Interface").

Availability. Copies of this paper, a descriptive source listing of the program CIRDIS, and documented output from an illustrative example are available at no cost from Dr. Wayne P. Aspey, Division of Comparative Neurobiology and Behavior, Marine Biomedical
Institute, University of Texas Medical Branch, 200 University Boulevard, Galveston, Texas 77550.

\section{REFERENCE NOTE}

1. Burgess, J. W. Personal communication.

\section{REFERENCES}

Barefoot, J. C., Aspey, W. P., \& Olson, J. M. Effects of partner novelty on affiliation in the rat. Bulletin of the Psychonomic Society, 1975, 6, 655-657.

Eckman, J., Meltzer, J., \& Latane, B. Gregariousness in rats as a function of familiarity of environment. Journal of Personality and Social Psychology, 1969, 11, 107-114.

KEMENY, J. G., \& KuRTZ, T. E. BASIC programming. New York: John Wiley. 1968.

LATANÉ, B. Gregariousness and fear in laboratory rats. Journal of Experimental Social Psychology, 1969, 5, 61-69.

Latané, B., Cappell, H., \& JoY, V. Social deprivation, housing density and gregariousness in rats. Journal of Comparative and Physiological Psychology, 1970, 70, 221-227.

LAtANÉ, B., \& Glass, D. C. Social and non-social attraction in rats. Journal of Personality and Social Psychology, 1968, 9. 142-146.

Latané, B., Schneider, E., Waring, P., \& Zweigenhaft, R. The specificity of social attraction in rats. Psychonomic Science, 1971, 23, 28-29.

Nevison, J. M. Computing in the liberal arts college: Science, 1976, 194, 396.401.

SHARPE, W. F. An introduction to computer programming using the BASIC language. New York: The Free Press, 1967. 